\title{
Coopétition et brevets : enjeux juridiques
}

\section{Coopetition and patents: legal stakes}

\author{
Nicolas Binctin ${ }^{1}$ \\ ${ }^{1}$ Université de Poitiers, CECOJI, France, nicolas.binctin@univ-poitiers.fr
}

RÉSUMÉ. L'intégration des brevets dans l'organisation de la coopétition entre opérateurs économiques est une question complexe. Appelant un environnement spécifiquement adapté à une telle pratique, la coopétition, encouragée mais aussi crainte sous l'angle du droit de la concurrence, suppose que les opérateurs adaptent leur stratégie de brevet. Le premier outil mobilisé est le contrat destiné à encadrer la coopétition. La liberté contractuelle et la souplesse de la propriété intellectuelle sont exploitées pour permettre d'atteindre des objectifs en apparence opposés. La coopétition conduit aussi les opérateurs à développer des analyses renouvelées de la gestion de leurs actifs intellectuels, passant d'une approche exclusive à une proposition de partage, sans perdre l'intérêt économique de ces actifs. II est possible de retenir trois pratiques qui placent la coopétition comme outil de gestion : l'appréhension des cycles technologiques, la gestion de portefeuilles de brevets pour porter la croissance du marché et, enfin, le corporate venture.

ABSTRACT. The integration of patents in the coopetition dynamic between economic operators is a complex issue. Calling on an environment specifically adapted to such a practice, coopetition, encouraged but also feared by competition law, assumes that operators adapt their patent strategy. The first tool mobilized is the contract intended to supervise coopetition. Contractual freedom and IP flexibility are used to achieve seemingly opposite goals. Coopetition also leads operators to develop renewed analyses of the management of their intellectual assets, moving from an exclusive approach to a sharing proposal, without losing the economic interest of these assets. It is possible to retain three practices which place coopetition as a management tool: understanding technological cycles, managing patent portfolios to drive market growth and, finally, corporate venture.

MOTS-CLÉS. Propriété intellectuelle, Brevet, Stratégie, Coopétition, Ecosystème, Liberté contractuelle, Portefeuille de brevets, Cycles technologiques, Corporate venture.

KEYWORDS. Intellectual property, Patent, Strategy, Coopetition, Ecosystem, Contractual freedom, Patent portfolio, Technological cycles, Corporate venture.

\section{Introduction}

La question de la coopétition / innovation ouverte relève certes des usages partagés mais aussi du partage de compétences au stade de la création ${ }^{1}$. La propriété intellectuelle est une question juridique aval de l'acte créatif, mais elle ne peut pas totalement être indifférente à cet acte. Pensée historiquement pour une activité créative individuelle, la propriété intellectuelle est confrontée à une évolution des pratiques créatives. Ces pratiques influencent la mise en œuvre de nos outils juridiques. Ainsi, le développement de l'innovation au sein d'une entreprise est de plus en plus le fruit d'une collaboration avec des tiers. L'entreprise, qui n'a pas l'ensemble des ressources humaines, des expertises, nécessaires en interne, doit collaborer avec des ressources externes. L'entreprise peut, en outre, rechercher des partenaires pour, en plus de leurs compétences, partager les risques de l'innovation et ainsi ne plus assumer seule l'ensemble des coûts de la création. La coopétition trouve ainsi son fondement dans deux éléments complémentaires : la recherche de compétence et le partage du risque.

La collaboration avec des partenaires extérieurs est usuelle, elle prend la forme de l'intervention du laboratoire d'une université, ou encore d'un designer produit. On la retrouve potentiellement à tous les stades de développement du projet pour combler les carences des ressources internes et optimiser l'utilisation de celles-ci. À la demande de l'initiateur, le tiers crée des biens intellectuels

\footnotetext{
${ }^{1}$ L'open innovation est un moyen de collaboration aboutissant, pour les opérateurs, à une situation de coopétition. Les notions se complètent mais ne sont pas synonymes.
} 
ou apporte ses compétences pour permettre la création de biens intellectuels. La sous-traitance ou le recours à des compétences externes ne conduit pas à une externalisation complète ; il faut être à même de construire le programme de développement, le suivre et le réceptionner. Il est nécessaire d'avoir en interne les compétences humaines pour réaliser, à tout le moins, ces tâches. L'expertise spécifique est entre les mains du sous-traitant mais l'expertise globale et l'intégration des éléments développés en externe restent du ressort du donneur d'ordres ou de chacun des opérateurs lancés dans une démarche de coopétition. Si l'innovation prendre la forme d'un co-développement, les équipes travaillent ensemble, dans une approche assez similaire à celle de la sous-traitance où interviennent au sein de l'équipe de l'entrepreneur des experts venant ponctuellement répondre à un besoin.

Cette collaboration créative peut être délicate à organiser lorsque les partenaires qui collaborent ont des cultures et des attentes différentes. Par exemple, le besoin d'une solution rapide et exploitable en production est parfois complexe à mettre en œuvre dans un environnement académique de recherche. La négociation de contrats entre des opérateurs soumis aux contraintes du droit public avec des entreprises de droit privé n'est pas plus évidente. L'ajout d'éléments d'extranéité ne simplifie pas d'avantage la négociation. Pourtant, on note que les Etats dont les PME collaborent le plus avec la recherche universitaire sont les Etats où l'innovation est la plus dynamique [OCD 15]. Le mouvement de collaboration innovante est un mouvement profond de la gestion de la créativité dans l'entreprise. Plusieurs éléments de contexte expliquent cette volonté d'ouverture :

- la multiplicité des savoirs et des compétences nécessaires et la difficulté de tous les maîtriser en interne, en particulier lorsqu'ils ne sont pas liés au cœur de métier ;

- l'internationalisation des marchés et la nécessité croissante d'adapter les services produits aux usages locaux ;

- l'hyperspécialisation de certains marchés sur lesquels se multiplient des acteurs de niche plus spécialisés, plus réactifs, plus innovants ;

- la nécessité de développer une agilité forte dans des marchés incertains, notamment ceux à forte composante technologique, qui pousse à intégrer des composants externes complémentaires pour répondre au plus près de la demande.

Les enjeux juridiques de ces pratiques, s'ils doivent ici être abordés à l'aune du droit de la propriété intellectuelle, se retrouvent aussi en dehors de cette matière. Le droit de la concurrence, en premier lieu, apporte une attention toute particulière à ces collaborations d'un genre spécial entre concurrents. Les règlements d'exemption par catégorie viennent tout à la fois encourager et encadrer ces pratiques trouvant une voix originale face, à la fois aux contraintes des ententes et celle des aides $\mathrm{d}^{\prime}$ 'Etat. On pense au règlement d'exemption accompagnant le co-développement ${ }^{2}$, mais aussi celui visant la co-production ${ }^{3}$, et enfin, le très sensible règlement sur le transfert de technologie ${ }^{4}$. L'UE est aussi un acteur actif de ce co-développement grâce à ses programmes de financement de ce type de pratiques innovantes, les PCRD, dont le H2020, impose une collaboration créative entre des institutions de différents pays.

Après avoir envisagé les enjeux de la coopétition au stade de la création, nous les envisagerons au stade de l'exploitation.

\footnotetext{
${ }^{2}$ Règlement 1217/2010.

${ }^{3}$ Règlement 1218/2010.

${ }^{4}$ Règlement 316/2014.
} 


\section{Les enjeux au stade de la création de biens intellectuels}

Le droit accompagne la coopétition au moins sous deux angles au stade de la création de biens intellectuels. Ces éléments peuvent certainement être complétés par d'autres facteurs, mais il semble que ceux-ci sont essentiels pour l'existence d'un mécanisme, et plus encore, d'une culture de la coopétition. Il faut, en premier lieu, un écosystème (A) où les coopérants peuvent travailler ensemble, s'identifier, se connaître, échanger en toute liberté. Il faut, ensuite, un droit des contrats ouvert (B), adaptable, et la réforme du droit des obligations a très sensiblement contribué à cet aspect.

\subsection{A la recherche d'un écosystème créatif}

La coopétition suppose un environnement adapté, un écosystème créatif, par nature rare et fragile. On en connait les ingrédients : des formations supérieures de haut niveau, des entreprises de toutes tailles qui collaborent ${ }^{5}$, des investisseurs ayant une culture du risque, des financements publics importants, un marché ouvert et fort. Ces lieux sont rares, on pense naturellement à la Silicon Valley $^{6}$, on peut ajouter sans aucun doute la tech Valley de Tel-Aviv, probablement aussi l'écosystème en pleine croissance entre Shenzen et Canton. Les retombées des recherches menées par Stanford généreraient $55 \%$ du chiffre d'affaires de 150 entreprises de la Silicon Valley. L'Europe n'a jamais été capable de développer un tel environnement, malgré trente années de politiques publiques en quête de ce graal. L'Inde, pays pourtant riche en ressources humaines compétentes dans les nouvelles technologies connaît une difficulté similaire. En l'absence de cette réelle alchimie, l'Inde n'a pas non plus été en mesure de créer des leaders mondiaux d'internet ou des technologies du mobile.

Au-delà d'une proposition doctrinale et d'un effet de mode, l'open innovation est un mouvement ancien de gestion de l'innovation et répond à des situations très concrètes. De très nombreux travaux sont produits depuis quinze ans sur ce sujet, notamment le très complet et didactique rapport de l'Institut Open Innovation qui guide les développements suivants ${ }^{7}$. On peut identifier trois piliers théoriques pour cette approche de l'innovation.

En premier lieu, le livre fondateur de Chesbrough, Open innovation: The new imperative for creating and profiting from technology [CHE 03], et sa représentation de ce qu'est l'open innovation. Ce travail de systématisation a permis une forte diffusion de cette pratique notamment après des étudiants des grands MBA de la planète, qui ont ensuite diffusé dans leurs entreprises.

En deuxième lieu, il y a la notion de capacité d'absorption d'une entreprise développée par Cohen et Levinthal [COH 90] dans un article intitulé Absorptive capacity: A new perspective on learning and innovation. Suivant ces auteurs, la capacité d'absorption d'une entreprise est constituée par «la capacité d'une entreprise à reconnaître, à assimiler ou transformer, et à appliquer en interne des savoirs externes. Cette notion inclut également la capacité qu'a une entreprise à s'adapter (voire à se réorganiser) pour pouvoir absorber (utiliser) des savoirs provenant de l'extérieur». L'intégration de cultures extérieures est une des opérations les plus délicates à gérer pour l'entreprise. La coopétition ne doit pas emporter la dislocation des ressources internes mais les renforcer. Le développement d'une culture d'entreprise adaptée est un enjeu central, il en va de même pour la culture académique, ce n'est pas l'un contre l'autre mais l'un avec l'autre.

\footnotetext{
${ }^{5}$ Voir la description concrète de l'expérience de Julia (2019) dans la Silicon Valley, pp. 34-94.

${ }^{6}$ On constate que malgré la présence notamment du MIT et de la puissance financière de New-York, la Côte Est n'a pas été à même de développer le même écosystème.

${ }^{7}$ Rapport de Servajean-Hilts (2014), disponible à l'adresse www.institutopeninnovation.fr. 
Enfin, en troisième et dernier lieu, il y a la notion de lead-user [VON 86]. Le lead-user désigne l'utilisateur d'un produit ou d'un service qui présente deux caractéristiques. D'une part, il est à l'avant-garde d'une nouvelle tendance et, d'autre part, il a un fort intérêt personnel à trouver des solutions pour répondre aux attentes qui naissent (ou naîtront) de cette tendance. Les lead users imaginent et développent, avant la masse, des solutions qui répondent à des attentes qui se généraliseront par la suite à l'ensemble des utilisateurs du même domaine. Aussi, une entreprise a tout intérêt à savoir identifier et s'appuyer sur cette catégorie d'utilisateurs pour développer son innovation, que ce soient des individus ou des communautés d'utilisateurs. En pratique, l'exemple le plus évident est celui de l'iPhone 1, pour lequel Apple utilisa des technologies onéreuses et non encore exploitées par leur propriétaire pour les intégrer dans son design et apporter une nouvelle offre sur le marché. Les autres déclinaisons de la gamme n'ont en rien répondu à l'effort du premier de la liste.

La mise en place par une entreprise d'une politique d'Open Innovation impose, pour celle-ci, de dépasser une importante barrière culturelle. Elle doit passer d'une culture «fermée » à une culture « ouverte », changer de culture de gestion de la confidentialité, répartir les nouvelles fonctions entre les différents départements de l'entreprise, et adapter sa politique de ressources humaines aux personnels fortement impliqués dans l'Open Innovation ${ }^{8}$. La réussite de l'acquisition de savoirs externes par le biais d'une stratégie d'Open Innovation suppose pour l'entreprise de savoir effectuer la recherche de savoirs externes, leur intégration en interne puis leur commercialisation. La pratique de référence usuellement citée pour identifier la mise en œuvre d'un programme d'Open Innovation par une entreprise est l'exemple de Procter \& Gamble avec le lancement en 1999 de son initiative Connect and Develop ${ }^{9}$. Cette société développe son expertise interne et emploie plus de 9000 chercheurs dans quelque 27 centres de recherche et développement, mais s'appuie également sur des idées et inventions de l'extérieur : $42 \%$ des produits de Procter \& Gamble intègrent un élément majeur provenant de tiers.

Dans la perspective d'une entreprise pratiquant l'Open Innovation, deux modalités se dégagent : la modalité outside-in (ou inbound) et la modalité inside-out (ou outbound). L'Open Innovation outside-in signifie qu'une entreprise cherche à trouver des connaissances, méthodes ou technologies à l'extérieur pour enrichir ses propres processus d'innovation. Dans le cas de l'Open Innovation inside-out, elle porte ses connaissances, ses actifs intellectuels, à l'extérieur, la plupart du temps sous forme de licences, notamment suivant des modèles libres. Dans sa matérialisation économique, cette stratégie se traduit par des plates-formes et outils d'échanges de type Give and Get (donner/recevoir) ou des plates-formes mutualisées d'innovation.

Dans l'une ou l'autre de ces approches, l'Open Innovation peut s'envisager de deux façons. D'une part, il existe le mode «pull» où une entreprise cherche à répondre à une problématique plus ou moins précise mais identifiée. La recherche ou l'identification de nouvelles sources de savoir peut être réalisée à travers différents canaux, auprès de différents acteurs, depuis l'appel à la foule jusqu'à l'identification de spécialistes portant des savoirs très spécifiques. D'autre part, on observe le mode «push» où une entreprise accueille toutes les idées venant de l'extérieur : une «boîte à idée » ouverte aux acteurs externes et non plus seulement à ses employés.

Il ressort de l'essentiel des travaux que le processus créatif fondé sur une approche par l'open innovation suppose de réunir au moins cinq éléments clés :

- Le réseau ;

\footnotetext{
${ }^{8}$ Voir Servajean-Hilts, rapport, op. cit. Nbp7, p. 15.

${ }^{9}$ Voir OMPI Magazine, $\mathrm{n}^{\circ}$ 6, Décembre 2012, p. 12 et sq., disponible à l'adresse

https://www.wipo.int/wipo magazine/fr/2012/06/
} 
- La collaboration, aussi bien avec les partenaires que les concurrents, les universitaires, et les usagers ;

- Une culture d'entreprenariat (Corporate entrepreneurship) alliant start-up, spin-offs et le corporate venturing ;

- Une gestion dynamique de la propriété intellectuelle pour faire émerger de nouveaux marchés pour les innovations ;

- Une R\&D active fournissant des avantages compétitifs.

L'innovation est conduite par l'intégration de la collaboration, le partage des valeurs de la création conjointe, le développement d'un écosystème innovant, le développement exponentiel des hautes technologies, et une extrême rapidité de leur diffusion. Cette idée est celle d'une massification des sources de l'innovation, en opposition à une approche actuelle assimilée à un art maîtrisé par peu. Cette approche est intéressante, elle n'en demeure pas moins tintée d'une certaine utopie. La mise en œuvre de ces logiques peut influencer l'organisation des opérateurs autant pour un co-développement externe, image classique, qu'interne.

Pour le co-développement externe, le sujet est assez connu à ce jour. Pour la propriété intellectuelle, le développement de la recherche collaborative déplace la frontière entre secret et brevet. En effet, plus l'entreprise ouvre et partage ses ressources pour mieux innover, plus elle doit organiser l'appropriation de ces dernières. Le secret, un temps privilégié peut laisser sa place aux brevets. Monsieur Bauvir, responsable du service propriété intellectuelle du groupe Michelin, explique que l'entreprise a dû adapter sa politique de propriété intellectuelle à ses programmes de recherche collaborative ${ }^{10}$. Ainsi, la stratégie de la société consiste à utiliser les brevets comme régulateurs des droits respectifs des différents partenaires. Par exemple, la société a conclu un accord-cadre avec le CNRS, pour la France, qui facilite l'accès à la coopération avec des UMR. Il s'agit de déposer des brevets qui respectent les droits respectifs de chaque partie, au regard des contributions créatives de chacune d'elle, de sorte à instaurer de véritables partenariats. Cette stratégie a conduit le groupe à breveter de plus en plus d'innovations. De 120 brevets par an au tournant des années 2000, l'entreprise est passée à 400 délivrés par an. En pratique, dans le cas de ses programmes de recherche partenariale, Michelin dépose d'abord des brevets de base, puis, lorsque la coopération donne ses fruits, la société dépose des brevets supplémentaires au fil de l'eau. Cette démarche tranche quelque peu avec la réputation de discrétion, voire de culture du secret, du groupe. Les pratiques évoluent, d'une part, avec l'influence de la rétro-ingénierie et de l'intensification des coopérations internationales de recherche, et, d'autre part, avec le développement d'activités commerciales dans la quasi-totalité des pays du monde. Si le secret continue de faire partie de la stratégie de Michelin, la frontière entre secret et brevet se déplace de plus en plus vers le second. La société est guidée dans sa stratégie par le pragmatisme : elle recherche des brevets pour les innovations qui se voient et garde secret ce qui ne se voit pas. Dans ce contexte, le recours aux mécanismes du secret d'affaires semble plus secondaire.

La force de l'innovation collaborative est qu'elle peut aussi conduire à repenser l'organisation interne de l'innovation d'une entreprise. La tertiarisation et la numérisation de l'entreprise explique cette tendance et en constitue souvent un moteur de la transformation interne. Par exemple, la société Vinci a ouvert le centre commun de recherche Leonard, à Paris, qui mutualise l'innovation entre ses différents métiers, des concessions autoroutières au BTP en passant par les services à l'énergie et l'immobilier alors que ces derniers n'innovaient guère ensemble. Il y a là un exemple de coopétition/innovation collaborative interne particulièrement intéressant. Ce centre fournit à la société un lieu de réflexion transversale, ouvert sur les start-up et tout l'écosystème extérieur. L'entreprise peut conduire une réflexion transversale sur la mobilité du futur (véhicule autonome, le

\footnotetext{
${ }^{10} \mathrm{https://www.inpi.fr/fr/valoriser-vos-actifs/le-magazine-de-la-valorisation/secrets-ou-brevets-la-reponse-de-michelin}$ https://www.lesechos.fr/2018/06/michelin-a-triple-ses-depots-de-brevet-en-quinze-ans-973644
} 
péage autoroutier sans barrière etc.), ou encore la ville de demain, sa logistique face au boom du ecommerce et sa densification.

\subsection{Les enjeux contractuels}

L'organisation d'un processus de coopétition impose une maîtrise des outils contractuels très poussés, avec une réelle capacité de se projeter dans l'organisation d'une conduite de projet, marquée par la présence de forts aléas liés à toute opération de création, d'innovation. La question de la propriété intellectuelle est sensible, mais n'est pas la seule. Dans certaines hypothèses, un tiers créateur peut être propriétaire des biens intellectuels créés à la demande ou en partenariat avec l'entreprise. Il est nécessaire de parvenir à savoir quels sont les enjeux centraux et ceux plus secondaires de l'entreprise, les biens intellectuels pour lesquels la propriété est impérative, ceux pour lesquels un droit d'exploitation, simple ou exclusif, répond pleinement à ses besoins et attentes. Il faut identifier les éléments recherchés grâce à la coopétition, les livrables dans le vocabulaire pratique, et s'assurer, par le biais des conventions, la propriété ou la jouissance de ces éléments, selon ses besoins. La logique est très proche de celle d'une association temporaire, d'une société de projet. Le réel enjeu pour les parties industrielles, plus que la propriété, est la liberté d'exploitation... La coopétition impose de passer d'une accumulation brute d'actifs intellectuels à une gestion dynamique et opérationnelle de ceux-ci.

Au regard des enjeux juridiques de la gestion de la recherche collaborative, au-delà de la seule question $\mathrm{du}$ partage des droits de propriété intellectuelle [SAU 2012], l'intégration du pluriculturalisme est le plus délicat. La stratégie juridique suppose de comprendre les cultures juridiques des différents opérateurs, parties à la collaboration de recherche. D'un point de vue structurel, le contrat de collaboration de recherche a un volet innovation établi par les personnels créatifs des opérateurs et un volet juridique établi par les juristes. Si la culture de l'innovation est très transfrontalière, la culture juridique ne l'est guère. Organiser une convention devant réunir des opérateurs publics et privés de plusieurs États, aux langues différentes et aux cultures juridiques fortes constitue un enjeu important [GUI 95]. L'analyse des contrats de consortium proposés pour les programmes de collaboration de recherche financés par l'Union européenne en est la preuve. Si les dispositions substantielles n'appellent guère de spécificité, la question de la loi applicable au contrat, ou celle de la juridiction compétente pour trancher d'éventuels conflits, fait ressortir ces différences [VER 18], [DEL 16], [BRO 15].

Le partage de la propriété des biens intellectuels entre coopétiteurs est le fruit de mécanismes contractuels. Il peut être fait selon les champs d'activité, il peut aussi être convenu qu'ils exploitent ces biens concurremment... parce qu'ils sont en compétition grâce à d'autres éléments de leurs produits et services. Le contrat de partenariat doit, non seulement, décrire la prestation à accomplir, mais aussi anticiper le sort des biens, des créations, issus de ce travail. Cette projection vers le futur est d'autant plus compliquée que la nature des livrables peut être difficilement identifiable. En pratique, il semble qu'il y ait, pour la recherche appliquée, peu de difficultés contractuelles. Les parties savent ce que les autres peuvent apporter et organisent le contrat pour partager les résultats afin d'en exploiter pleinement les avantages du travail commun. L'accès à des compétences ou des savoir-faire complémentaires est le premier objectif affiché par les opérateurs s'engageant dans une démarche de coopétition pour innover. L'intérêt d'une copropriété peut être longuement discuté, tout comme le recours à des démembrements réels conventionnels. La fiducie gestion, entendu comme un mécanisme de transfert de propriété par un constituant vers un fiduciaire, qui tient les éléments reçus séparés du reste de son patrimoine, pour les gérer dans un but précis déterminé par le contrat de fiducie, au profit d'un bénéficiaire ${ }^{11}$, qui peut éventuellement être le ou les constituants, peut aussi être une voie complémentaire pour approprier et gérer le fruit de la coopétition. On peut

${ }^{11}$ Voir art. 2011 C. civ. 
penser que les copropriétaires mettent les biens intellectuels communs entre les mains d'une fiducie gestion qui en assure la gestion au bénéfice de ces derniers.

Toutefois, le juriste évolue dans un environnement qui n'est pas naturellement le sien. Il doit passer d'une culture de l'anticipation absolue et du «zéro risque » emportant exclusion de toute incertitude, à une culture de contrat agile, de convention souple, adaptable au fur et à mesure de l'évolution des travaux, de la concrétisation de l'innovation. Le contrat est un cadre de coopération et non plus une série de solutions figées et impératives. On arrête le principe d'une appropriation des résultats, mais on ne partage pas en amont les résultats. Cette idée d'agilité contractuelle conduit à remettre en cause les modèles contractuels figés, les solutions impératives. Elle impose aussi une réelle implication intellectuelle du juriste dans le projet pour en comprendre les enjeux, les attentes, les options de mise en application... Il se doit d'être un élément dynamique, porteur de solutions, de proposition, de capacité d'empathie. Le contrat structurant la coopétition est non seulement scruté à l'aune du droit de la concurrence mais doit aussi parvenir à trouver ses outils dans le droit civil et le droit des contrats d'affaires. La profonde intégration de la bonne foi dans les mécanismes contractuels, l'idée d'un équilibre des intérêts, trouvent heureusement un écho fort dans la réforme du droit des obligations.

Cette évolution de la relation contractuelle, cette agilité, permet la mise en avant de deux organes de la coopétition, d'une part, le comité de suivi et, d'autre part, du contract manager. Le premier organe, classique des contrats de collaboration de recherche, occupe un rôle de plus en plus important au fur et à mesure que la convention doit s'adapter lors de son application. Les parties délèguent à cet organe contractuel le pouvoir de mise en œuvre, d'adaptation, les éléments nécessaires à garantir l'agilité de la collaboration. Le rythme des travaux, les orientations de recherche, etc. peuvent être adaptés, il ne s'agit plus d'avenants ou de renégociations de contrat mais bien de phases d'exécution. Ce comité peut aussi établir les choix de propriété intellectuelle au regard des résultats, des attentes des parties, etc. Il doit, pour cela, compter non seulement des porteurs scientifiques du projet, mais aussi des experts métiers et des juristes. Il y a une déconcentration de la gestion de l'exécution du contrat. Le second organe qui émerge est le contract manager [SAV 16]. Si la prise de conscience de l'importance de la gestion du risque contractuel s'est développée depuis plusieurs décennies déjà [TUR 01, p.457], notamment dans les entreprises de construction, elle est bien plus récente pour les contrats portant sur des projets innovants. L'apparition de nouveaux modèles d'affaires, notamment la coopétition, et l'intensification des pratiques concurrentielles, ont complexifié les schémas contractuels et les contrats eux-mêmes. Dans ce contexte, les entreprises recherchent davantage de sécurisation des cycles de vie contractuels [LEV 13, p.218] ainsi qu'une optimisation de leur propre performance. Le Contract Management qui est par nature pluridisciplinaire, se nourrit de disciplines connexes : le droit, la gestion de projet, la finance, la sociologie, l'économie ou encore les sciences politiques. Ainsi, en plus de participer à la négociation et la rédaction du contrat, le contract manager suit l'application du contrat tout au long du projet, par la mise en œuvre et la coordination de moyens et processus nécessaires à la maîtrise des risques encourus et la réalisation d'opportunités potentielles.

Restent naturellement les questions classiques mais non moins importantes de tout contrat et qui demeurent complexes... La loi du contrat, le juge compétent... mais aussi le traitement de la variété des statuts des créateurs, la variété de ces statuts au regard des rattachements des créations/innovations aux catégories de la propriété intellectuelle. Il suffit ici de prendre simplement le cas d'une coopération européenne... chaque Etat propose une solution pour l'appropriation des créations de salarié pour chaque régime de propriété intellectuelle. Les combinaisons possibles rendent le modèle contractuel nécessairement risqué et le besoin d'un statut de créateur salarié européen impérieux ! 


\section{L'émergence de nouvelles pratiques}

La coopétition conduit les opérateurs à développer des analyses renouvelées de la gestion de leurs actifs intellectuels. On constate plusieurs phénomènes conduisant à une refonte de la gestion du portefeuille de brevets des opérateurs, passant d'une approche exclusive à une proposition de partage [EST 29], sans perdre l'intérêt économique de ce portefeuille. Il est possible de retenir trois pratiques qui placent la coopétition comme outil de gestion. Les logiques portées par ces pratiques sont complémentaires : l'appréhension des cycles technologiques, la gestion de portefeuilles de brevets pour porter la croissance du marché et, enfin, le corporate venture. Les développements suivants vont prendre principalement la forme d'illustrations pratiques, la mise en œuvre de celles-ci n'étant pas nécessairement juridiquement complexe. Toutefois, l'idée d'exploiter de la sorte un portefeuille de brevets constitue en soi une innovation.

\subsection{Cycles technologiques et portefeuille de brevets}

La question de l'adéquation entre les cycles technologiques et les portefeuilles de brevet est particulièrement complexe [LAN 15, p.34] et l'opérateur ne pouvant pas répondre à tous les marchés en même temps doit adapter son portefeuille à sa position sur le marché et à ces cycles. Ainsi, un opérateur portant une technologie premium pourrait se retrouver avec un portefeuille de brevets portant sur des biens intellectuels occupant toujours une place importante sur le marché mais ne répondant pas à sa propre stratégie de marché. Il est naturellement envisageable de vendre... mais au risque de se retrouver dans une situation de dépendance à l'égard de l'acquéreur.

Pour faire face à ce dilemme, IBM a totalement intégré la stratégie Open Innovation dans son modèle de développement, tout en conservant un modèle propriétaire fort sur ses avantages compétitifs essentiels. On peut prendre pour exemple deux programmes de cette société.

En premier lieu, la société est l'un des premiers contributeurs au financement du développement du système d'exploitation Linux pour lequel elle a investi plus de deux milliards de dollars en dix ans. Ces investissements ne sont en rien guidés par un souci philanthropique ; ils assurent des solutions technologiques efficaces pour la commercialisation d'autres produits, tels des microprocesseurs ou des solutions de serveurs et d'hébergements. La même logique existe pour le développement de logiciels afin de ne pas développer les lignes de code répondant à des fonctions récurrentes et sans valeur ajoutée. Par exemple, il s'agira du traitement des couleurs à l'écran, du traitement du clavier, de certaines interfaces, le réglage du son, etc. Ces développements sont nécessaires mais n'apportent pas un avantage concurrentiel, il est plus intéressant de collectiviser leur développement et d'en permettre un usage par tous. Les licences $L G P L$ ou $B S D$, par exemple, permettent sans grandes contraintes, la réutilisation de ce type de développements dans le cadre d'autres projets. Le rapport coûts/avantages est à nouveau au cœur de la stratégie de partage. Il ne s'agit pas de moins investir dans le capital intellectuel mais de mieux investir pour optimiser la constitution du portefeuille de biens intellectuels d'un opérateur. C'est un outil de concurrence pour s'émanciper notamment de Microsoft. Il y a partage des ressources logicielles ainsi développées, mais le rapport coûts/avantages est bénéfique pour la société qui partage les coûts avec les autres contributeurs, tel Oracle, et assure des marchés pour ses autres produits en maintenant une indépendance technologique.

En second lieu, IBM a initié le projet OpenPOWER Consortium en 2013. Dans ce cadre, IBM intègre dans une stratégie Open Innovation l'ensemble de sa technologie POWER, dont les spécifications des processeurs, les firmware et les logiciels afin de permettre un meilleur développement collaboratif de cette activité. L'objectif est de créer un nouvel écosystème permettant d'adapter la commercialisation de serveurs aux nouvelles exploitations et attentes des utilisateurs, notamment pour le cloud computing et les data centers. Ce projet implique notamment Google, Samsung, Tyan, Nvidia, Mellanox, Altera, Fusion-io, Hynix, Micron, Servergy, Xilinx, 
Canonical, Chuanghe Mobile, Emulex, Hitachi, Inspur, Jülich Research Centre, Oregon State University, Teamsun, Unisource Technology Inc ou encore ZTE. Aucun de ces opérateurs ne renonce à son propre modèle de développement mais connaît par cette stratégie un moyen de partager le risque et de développer des solutions que, seuls, ils auraient été en peine de développer technologiquement et financièrement. IBM continue de construire ses serveurs Power mais concentre ses efforts d'exploitation/commercialisation uniquement sur la dernière génération de serveurs. En parallèle, $I B M$ enrôle d'autres fournisseurs pour l'aider à gagner des clients du côté des fournisseurs de services en ligne à grande échelle aux États-Unis et en Chine. Ces entreprises achètent de nombreux serveurs, mais souhaitent des machines très adaptées, peu coûteuses et sans fioritures. C'est un modèle différent de celui $I B M$. En instaurant le consortium, $I B M$ poursuit sur son marché premium et occupe le marché second au travers de l'alliance de fournisseurs.

Microsoft a eu aussi à faire face à cette difficulté. Ainsi, l'Open Innovation Network, lancée en 2005, a bénéficié de financement de la part, notamment, de IBM, NEC, Novel, Philips, Red Hat ou encore Sony. Ces sociétés, fondées sur des modèles de propriété intellectuelle forts, ont contribué à créer Open Innovation Network afin de promouvoir le système Lynux en utilisant leurs brevets pour enrichir la création collaborative dans cet environnement technique et légal spécifique. Open Innovation Network tente d'articuler le cadre légal des logiciels libres et le droit des brevets. Les inventions issues de ces recherches sont appropriées par brevets qui sont ensuite partagés dans un modèle libre. Les brevets appropriés par Open Innovation Network sont accessibles gratuitement pour toute société, institution ou personne qui acceptent de ne pas opposer ses brevets au système Linux $^{12}$. Les parties prenantes sont engagés pas leur souscription aux statuts et aux engagements de mise à disposition de leurs droits de propriété. L'engagement peut porter uniquement sur les brevets existants et remis, il peut aussi intégrer des brevets à venir. Les modèles contractuels sont assez variés [EST 19]. Cette démarche permet à ces sociétés de réaliser de substantielles économies d'échelle en partageant des coûts de développement au travers du modèle contractuel libre. Microsoft a annoncé en octobre 2018 rejoindre l'Open Innovation Network et accorder aux membres du groupement des licences sur tous ses brevets en lien avec le système Linux, soit environ 60000 brevets. L'usage envisagé est pleinement stratégique, il doit notamment permettre aux mêmes du groupement de limiter les risques d'action en contrefaçon. Les licences entre les membres sont gratuites. Cette participation montre que Microsoft a fait évoluer sa stratégie de propriété intellectuelle sans pour autant renoncer à la propriété intellectuelle. La société en adapte ses formes d'exploitation à l'évolution du marché. La suite Office est moins fondamentale pour l'entreprise que son offre de service en cloud...Par ailleurs, Linux semble largement retenu pour l'Internet des objets ce qui pourrait avoir encouragé la société à s'intégrer dans le mouvement pour s'assurer une parfaite compatibilité de ses produits avec cette technologie.

\subsection{Gestion de portefeuilles de brevets et croissance du marché}

L'Open Innovation permet un mouvement dynamique de la gestion d'un portefeuille de biens intellectuels. Le cadre est assez simple, un opérateur dispose de ressources données pour développer ses produits mais le coût de développement est supérieur à celles-ci. De plus, une partie des développements devant être réalisés ne lui apportera qu'un très faible avantage concurrentiel. Le rapport entre le coût de développement et le rendement de leur exploitation est assez faible. Dans ce cadre, il peut être intéressant d'envisager de partager les coûts et les risques de développement avec des tiers, même si, en conséquence il y a un partage des biens intellectuels créés. Si le modèle de la copropriété pourrait s'appliquer à cette situation, des modèles de licences ouvertes sont utilisés pour assurer l'accès aux biens intellectuels développés collectivement [CLE 14]. A la différence de la copropriété, les modèles de licences ouvertes laissent chaque partie propriétaire de sa contribution, le contrat de licence assurant l'articulation des différentes contributions, différents biens

\footnotetext{
${ }^{12}$ http://www.openinventionnetwork.com/pat_license_agreement.php.
} 
intellectuels, entre eux et les conditions d'exploitation du bien intellectuel issu de la combinaison. Ainsi, le recours à la copropriété apparaît adapté lorsque les partenaires sont connus et limités, et que les parties souhaitent conserver une maîtrise de l'exploitation du résultat. Le recours à un mécanisme ouvert permettant l'intégration de communauté de développeurs/créateurs permet de mieux intégrer un modèle de développement où les partenaires potentiels ne sont pas identifiés ou identifiables, en raison de vastes applications possibles [BIN 16, p.185] ou en raison d'un mécanisme très incrémental de création. Rien n'interdit à des co-propriétaires d'un brevet de s'accorder pour mettre en place une licence ouverte pour l'exploitation de ce dernier, tout comme l'intégration dudit brevet au sein d'une communauté ouverte en autorisant l'exploitation de l'invention dans le cadre d'un ou plusieurs modèles de licences libres.

Dans le même esprit, pour porter la croissance d'un marché fondé sur une technologie, il est souvent nécessaire que plusieurs opérateurs puissent proposer cette dernière. A défaut, la dépendance est trop forte et détourne les entreprises de cette solution pour en rechercher une autre. Le porteur de la technologie la meilleure peut, au mieux, être alors leader... d'un micromarché. L'histoire de la propriété intellectuelle propose quelques exemples marquant de stratégie d'ouvertures de portefeuilles de brevets pour permettre une croissance du marché globale, plus bénéfique pour le propriétaire des brevets qu'une exploitation isolée de sa technologie. Cette pratique est parfois désignée sous le vocable de patent pledge [CON 15, p.543]. Le premier exemple est celui de la gestion par Michelin du pneumatique radial. Pour que le volume de pneumatiques fabriqués avec cette technologie croisse, au point d'être la seule aujourd'hui mise en œuvre, la société a partagé sa technologie [MIR 13, p.37]. Cet objectif de faire croitre le marché pour permettre une application à grande échelle de la technologie et de l'outil industriel se retrouve récemment à nouveau dans le secteur automobile, avec deux exemples pour des constructeurs radicalement différents. Le premier cas est l'initiative de Tesla [BIN 15], 2014, au travers de l'ouverture de son portefeuille de brevets sur la voiture électrique. L'enjeu pour la société est notamment de voire augmenter le marché de cette technologie pour croître elle-même et rassurer ses bailleurs de fonds. Le second cas est celui de Toyota ${ }^{13}$, en 2015, avec l'ouverture de son portefeuille de brevets portant sur la technologie du moteur à hydrogène. L'enjeu est le même pour la société, si elle est convaincue de la pertinence de cette solution, son déploiement à grande échelle suppose son adoption par d'autres opérateurs. Pour accélérer ce mouvement, le meilleur moyen reste de leur offrir la technologie. L'opérateur qui se lance dans une telle opération est aussi motivé par la conviction que sa maîtrise antérieure de la technologie lui laissera toujours un avantage compétitif sur les autres concurrents et qu'il conservera ainsi un temps d'avance sur le marché. C'est le cas de Tesla qui ouvre ses brevets mais pas ses logiciels. Ce fut aussi le cas de Michelin. Dans le cas de Toyota, le coût des infrastructures pour le déploiement en masse d'automobiles fonctionnant à l'hydrogène impose des partenariats vastes et une augmentation du nombre de véhicules en circulation intégrant cette technologie.

On peut se demander si la gestion des portefeuilles de brevets des institutions publiques de recherche ne devrait pas aussi s'inspirer de ces solutions. La valorisation de la recherche publique constitue une source importante, croissante, de biens intellectuels concourant au développement de l'actif intellectuel des entreprises. La valorisation de la recherche publique est aujourd'hui un enjeu important en Europe. L'OCDE insiste sur la nécessité pour les États d'avoir des politiques dynamiques en ce sens [OCD 13]. Les rapports de l'INPI, présentant les initiatives allemande [DOY 14], anglaise [INP 14a], israélienne [INP 14b] et américaine [DOY 14b] dans ce domaine, illustrent cette constante approche. L'enjeu est de fournir un débouché économique aux investissements publics dans la recherche, de permettre une meilleure pénétration des innovations les plus

\footnotetext{
${ }^{13}$ https://global.toyota/en/detail/4663648 voir aussi pour les véhicules hybrides, https://www.reuters.com/article/us-toyotapatents/toyota-to-give-royalty-free-access-to-hybrid-vehicle-patents-idUSKCN1RE2KC
} 
ambitieuses dans le monde économique et de fournir des ressources d'excellence aux entreprises. En France, les Instituts Carnot sont à l'origine d'une soixantaine de start-up par an.

La valorisation de la recherche publique est un processus associé à un horizon de temps assez long, notamment pour la recherche fondamentale et les technologies encore embryonnaires. L'expérience des universités américaines le confirme. Il a fallu plus de treize ans pour établir la première vague des bureaux de valorisation issus de loi Bayh-Dole, et plusieurs années supplémentaires pour les doter du personnel qualifié requis et développer leur culture professionnelle. A partir d'un portefeuille de créations, un à quatre ans sont nécessaires pour trouver un entrepreneur susceptible de s'engager à les développer et les exploiter. L'enjeu de la valorisation de la recherche publique n'est pas d'accroître les ressources propres des établissements supérieurs de recherche. Les revenus dégagés par ce biais contribuent à ces ressources de façon marginale [LAL 13, p.6] L'enjeu est de permettre une meilleure mutualisation des investissements publics dans le développement des connaissances et de permettre aux opérateurs privés de bénéficier des acquis des organismes publics de recherche. Dans cette logique, la valorisation de la recherche publique peut aussi prendre la forme d'une ouverture globale des connaissances. On peut penser au mouvement open data [BIN 15], on peut aussi évoquer la politique de l'Université de Stanford qui propose dans certains cas à ses chercheurs le choix entre l'appropriation et le domaine public pour certaines de leurs créations s'ils estiment que cela permet une meilleure diffusion du savoir en cause. Cette université est porteuse d'un modèle de valorisation de la recherche particulièrement vertueux, encourageant les étudiants à l'entrepreneuriat et en fournissant des moyens d'accompagnement des projets. Elle a accueilli les fondateurs de Google qui étaient inscrits en thèse, ceux de Yahoo!, Hewlett Packard et celui de Nike. Berkeley a, pour sa part, porté l'invention du langage Pascal et a accueilli un des fondateurs d'Apple et celui du Sun Microsystem. Ces institutions d'enseignement et de recherche ont su développer une culture spécifique pour accompagner les projets de leurs étudiants.

\subsection{Les fonds d'investissement d'entreprises - corporate venture -}

Le dernier mouvement engagé par les entreprises pour permettre d'attirer à elles les innovations qu'elles ne seraient pas à même de faire émerger en leur sein prend la forme de fonds d'investissement. Ce capital-risque d'entreprise doit se distinguer de stratégies d'investissements purement financières. Il s'agit d'accéder à la technologie plus que de rechercher des rendements à court terme sur les fonds investis. Il ne s'agit pas pour autant de philanthropie. Cette approche des actifs intellectuels émerge depuis 2010 dans la stratégie des entreprises, elle tend à prendre un essor important. Ces fonds accompagnent les entreprises dans leur perception des tendances de l'innovation, le plus en amont possible. L'approche par le financement est une démarche originale d'identification des innovations, au lieu de les cibler par le truchement du département R\&D. Pour l'entreprise, la quête du rythme du marché est difficile : plus l'entreprise est grande, plus elle risque de s'éloigner des tendances les plus en pointe, en raison de sa perte d'agilité. Le fonds d'entreprise permet d'offrir un autre accès à cette dynamique. Les fonds recherchent autant les innovations technologiques que celles de services ou de modèles économiques. L'entreprise, sans exclure à terme les opérations sur capital ni la recherche collaborative et la conclusion de contrats de concession, souhaite permettre aux jeunes entreprises innovantes de pouvoir porter à maturité leurs créations. Il n'y a pas d'opération aval possible pour intégrer ces créations tant qu'elles ne sont pas finalisées, fiabilisées. Les fonds d'entreprise ont vocation à apporter des ressources financières à des entreprises portant des créations à haut potentiel et qui recherchent des investissements pour finaliser leur développement. Pour les investisseurs, il est évident que cela constitue une autre voie d'accès aux biens intellectuels, plus médiate, plus en amont des solutions antérieures. Cet accès se fait, d'une part, par un droit de regard sur les solutions technologiques accompagné d'une obligation de confidentialité et de non-concurrence, et, d'autre part, sur une possibilité de proposer un partenariat, en général non-exclusif, dans des conditions de marché. Le fonds d'investissement 
organise un partage du risque, il n'est pas seul à porter l'innovation. Pour l'entreprise investisseur, le rapport coût/risque est attractif. Pour le bénéficiaire de l'investissement, une telle opération permet non seulement de recevoir des fonds, mais aussi d'organiser un réseau d'entreprises pouvant soutenir, offrir des débouchés et construire un partenariat économique fort.

En 2018, plus de 2.740 tours de table pour un montant de plus de 53 milliards de dollars ont été investis suivant cette technique, soit une augmentation de $47 \%$ par rapport à $2017^{14}$. Le nombre d'opérations a également été multiplié par 5 depuis 2013. La pratique française accorde une place centrale dans cette évolution avec un nombre moyen d'investissements multiplié par 2 par rapport à 2016. Les secteurs de la mobilité et ceux des Cleantech/énergie ont attiré le plus d'investissements en 2018. A l'inverse, on constate une perte de dynamisme pour les secteurs de la Fintech/Insurtech et celui de la Healthtech. Dans une logique de synergie entre les grands groupes et les start-up, les Corporate Venture Capitalists (CVCs) français reconfigurent leurs stratégies d'investissement : 79\% de leurs investissements réalisés en 2018 ont répondu à un objectif d'Open Innovation, dans une démarche gagnant/gagnant.

Le Corporate Venture permet aux grandes entreprises de conserver leur capacité d'innovation au sein de leur marché et il donne accès à des fonds et à des savoir-faire externes en commercialisation et en gestion de projet à des petites structures porteuses d'un projet innovant. Pour les start-ups, le soutien financier et opérationnel d'un grand groupe est rassurant. Il valide la pertinence du projet et leur permet de poursuivre leur projet entrepreneurial dans les meilleures conditions d'autonomie pour leur développement. Le recours au CVC permet de raccourcir les phases de «go-to-market » des produits, un enjeu vital dans un univers où la vitesse est un facteur discriminant. Pour les grands groupes, le Corporate Venture répond à des objectifs stratégiques et organisationnels : veille concurrentielle, externalisation de la R\&D, etc. plutôt qu'à des investissements financiers classiques. Il est à penser avant tout comme un levier de transformation de l'entreprise qui permet de capter des innovations de rupture et aide à comprendre de nouveaux business models, de nouveaux usages ou des modes de consommation rendus possibles par la digitalisation. Cette pratique apporte une plus grande agilité et une capacité à pivoter plus rapidement pour s'adapter au marché. De plus, le risque est relativement limité : pas de changement de culture à mener et, si l'on arrive au bon moment, le modèle est déjà reconnu et son prix plus facile à anticiper (contrairement aux gros projets de recherche au budget plus incertain).

Dans cette pratique, les incompatibilités culturelles ne sont pas rares. Pour maximiser les chances de réussite, il convient, pour les jeunes pousses comme pour les grands groupes, de définir en amont les règles de la collaboration, notamment pour la propriété intellectuelle. L'esprit du CVC veut que l'intention du grand compte soit de contribuer au développement de la start-up sans s'engager dans une démarche de rentabilité purement opportuniste. La relation doit être fondée sur une confiance réciproque. Il faut que le CVC parvienne à résister à la tentation de diluer la start-up dans l'organisation historique. Il faut lui laisser son autonomie et sa liberté de manœuvre afin de préserver l'agilité des équipes, mais aussi parvenir à établir un rapport de coopétition.

La mise en œuvre de cette stratégie prend deux formes: soit plusieurs entreprises créent en commun un fonds d'investissement sectoriel, soit l'entreprise dispose de son propre fonds. Il n'est pas exclu, évidemment, qu'une entreprise participe aux deux mouvements. Pour le premier, Total, Renault, EDF ou Schneider cherchent ainsi à saisir les dernières tendances en matière d'innovation.

\footnotetext{
${ }^{14}$ Le troisième Baromètre Orange Digital Ventures et Deloitte, les investissements menés par des « Corporate Venture Capitalists » (CVC) https://www2.deloitte.com/content/dam/Deloitte/fr/Documents/services-

financiers/publications/deloitte barometre-cvc-2019.pdf; https://www.latribune.fr/entreprises-finance/banques-finance/lecorporate-venture-a-la-francaise-decolle-818021.html; https://business.lesechos.fr/directions-financieres/financement-etoperations/credits/0601384143748-les-corporate-francais-se-font-investisseurs-avertis-329830.php
} 
Engie, via son fonds New Venture Engie, un fonds de 100 millions d'euros, est entrée au capital de Redbird, un fabricant de drones, en investissant 2 millions d'euros. Ce fonds réalise des investissements dans des sociétés en lien avec les axes stratégiques de développement du groupe, notamment la production d'énergie décentralisée et le stockage, la gestion digitale de l'énergie, le confort domestique, les objets connectés, la ville et la mobilité. Dans le secteur financier, $A X A$ a créé un fonds doté de 200 millions d'euros en février 2015, un second a été créé en 2019 et l'ensemble est réuni sous la houlette de AXA Next qui tente de construire les métiers du futur pour l'assureur. Total, au travers de son fonds Total Energy Venture, détient des participations dans une vingtaine de jeunes entreprises innovantes pour un investissement de l'ordre de 150 millions de dollars. Selon cette société, la mutualisation des investissements représentée par l'action de ces sociétés représente l'équivalent de 2 milliards de dollars de dépenses de R\&D depuis 2008. Il n'est pas un secteur où la tendance n'est pas reprise. L'un des fonds les plus actifs est Google Venture, fonds créé par la société éponyme en 2009 pour investir dans les sociétés de nouvelle technologie. Doté de 100 millions de dollars à l'origine, ce fonds a investi dans une quarantaine de sociétés, dont la plus connue est Uber. Airbus Group Venture a installé un fonds de 150 millions en juin 2015, installé dans la Silicon Valley, en Californie. Le fonds initié par l'éditeur de logiciel SAP a connu un tel succès qu'il s'est dégagé de son initiateur pour prendre une activité plus autonome et changer de nom, devenant Saphire Venture.

L'utilisation de ces solutions conduit éventuellement à une véritable intégration industrielle, on pense au cas de Symbio, créée en 2010, avec une technologie obtenue grâce à une licence avec le CEA. Michelin est devenu actionnaire en avril 2014 dans le cadre de sa politique de Corporate Venture, avant d'en faire une filiale en février 2019, pour finalement créer une co-entreprise avec Faurecia en novembre de la même année. Selon un modèle similaire, la SNCF a placé son fonds de Corporate Venture au sein d'une structure dénommée 574 Invest. Cette dernière facilite les mises en relation avec les entités du groupe $S N C F$ pour à la fois développer l'activité commerciale de la startup, et accélérer des projets $S N C F$. Les participations peuvent s'accompagner de la mise en place d'accords commerciaux ou de développement entre les startups du portefeuille et une ou plusieurs entités de la $S N C F$.

Non seulement l'entreprise investit par ce biais, mais, plus encore, elle est en relation avec des entrepreneurs innovants. La prise de contact avec le marché compte autant pour suivre les tendances. Renault serait passé d'une cinquantaine de contacts par an avec des start-up grâce à un dispositif de veille, à environ 1000 contacts au travers de sa participation au fonds Partech. L'investissement s'accompagne le plus souvent d'un lien industriel, d'un échange de moyens technologiques. La présence d'un fonds intermédiaire qui lève des ressources auprès de plusieurs entreprises, suivant la stratégie de Renault, permet aussi un autre modèle original : les fonds publicsprivés.

Dans toutes les hypothèses, les montants investis restent mesurés face aux ressources des sociétés en question. Il s'agit par nature d'un investissement à risque pour lequel il est probable que neuf dixième des projets échoueront. L'enjeu est toutefois de comprendre les tendances et d'accompagner les $10 \%$ de projets qui connaitront un succès. L'entreprise s'ouvre, écoute, accompagne car elle ne pourra pas seule agir durablement et efficacement. L'accès aux innovations, aux créations est à cette condition. Il ressort clairement que l'innovation n'est plus du seul ressort d'une Fonction de l'entreprise, telle la Fonction R\&D, mais qu'elle mobilise toutes les compétences, toutes les ressources et pratiques. L'accès à l'innovation n'appelle pas nécessairement un accès à la propriété de celle-ci, en avoir la jouissance peut suffire maintenir une dynamique opérationnelle. 


\section{Conclusion}

L'utilisation de la propriété intellectuelle comme vecteur de la stratégie d'une entreprise doit devenir un réflexe ordinaire des managers. Le droit est flexible, il s'adapte, traduit les besoins et les objectifs des entreprises. Faut-il encore que les entreprises puissent identifier clairement ces derniers et que les juristes puissent les comprendre et les traduire dans les solutions juridiques. Le dialogue reste la clé d'une telle démarche.

\section{Références}

[BIN 15] BINCTIN, C., Stratégie d'entreprise et propriété intellectuelle, L.G.D.J, 352p, 2015.

[BIN 16] Binctin, C., The French copyright law opens its arms to the Free Software and Creative Commons, license contracts, free software and creative commons, Springer Science+Business Media, 185p, 2016.

[BRO 15] BRONZO, N., Propriété Intellectuelle et valorisation des Résultats de la recherche Publique, PUAM, 2015.

[BUR 11] Burger-Helmchen, T., Hussler, C., J. PÉNin, «General presentation. Rethinking boundaries for innovation: Exploring the shapes and stakes of the open innovation phenomenon », Journal of Innovation Economics \& Management, $\mathrm{N}^{\circ}$ 7, 2011.

[CLE 14 ] CLEMENT-FonTAINE, J-M., L'œuvre libre, Larcier, 2014.

[CHE 03] Chesbrough, H., «Open innovation: The new imperative for creating and profiting from technology », Harvard Business Review Press, 2003.

[CHE 03] Chesbrough, H., "Open business models: How to thrive in the new innovation landscape », Harvard Business Review Press, 272p, 2006.

[CHE 03] Chesbrough, H., Open Innovation : Researching a New Paradigm, Oxford University Press, 392p, 2008.

[COH 90] COHEN, W.M., D.A. LEvinTHAL, « Absorptive capacity: A new perspective on learning and innovation », Administrative Science Quarterly, 35, N , mars, 1990.

[CON 15] CONTRERAS, J., « Patent pledges », Azirona State Law Journal, Fall, vol. 47, Issue 3, 2015.

[DEL 16] DeLmotTE, A., Les aspects juridiques de la valorisation de la recherche, Mare et Martin, 958p, 2016.

[DOY 14] DOYEN, D., «Innovation collaborative et propriété intellectuelle - Les États-Unis », Analyses INPI, nov. 2014.

[EST 19] Esteves, N., Partager les brevets, étude des modèles ouverts en droit des brevets, Thèse, Sciences Po, 12 décembre, 2019.

[GUI 95] GuILlEmin, J-F., «Le tunnel sous la manche : Confrontation et fusion permanente de deux cultures juridiques réputés antagonistes », Revue Internationale de Droit Comparé, vol. 47, n², 403, 1995.

[JUL 19 ] JULIA, L., L'intelligence artificielle n'existe pas, First Edition, 200p, 2019.

[INP 14a] INPI, «Innovation collaborative et propriété intellectuelle - Le Royaume-Uni », Dossier n² 2014-1, janvier 2014.

[INP 14b] INPI, «Innovation collaborative et propriété intellectuelle - Israël », Dossier n 2014-3, juillet 2014.

[LAL 13] LALLEMENT, R., "Valorisation de la recherche publique : quels critères de succès en comparaison internationale? », La note d'analyse Économie-Finances, Centre d'analyse Stratégique mars, n 325, 2013.

[LAN 15] LANTZ, J-S., « Gestion dynamique des portefeuilles de brevet et création de valeur », Journal des Sociétés, $\mathrm{n}^{\circ} 130$, mai, 2015.

[LEV 13] LeVeau, G., Pratique du contract management - Optimisez la gestion du cycle de vie contractuel, Gualino Lextenso éditions, 224p, 2013.

[MIR 13] MiRATON, D., «Comment Michelin a ouvert son innovation», Le journal de l'école de Paris du management, $\mathrm{N}^{\circ} 99,2013$.

[OCD 13] OCDE, « Nouvelles sources de croissance : le capital intellectuel », Rapport, mai, 78p, 2013.

[OCD 15] OCDE, «The innovation imperative - Contributing to productivity, growth and well-being », Rapport, Octobre 2015. 
[QUO 15] QUONIAM, L., L'innovation frugale, disponible à l'adresse http://www.marseille-innov.org/frugalinnovation-une-demarche-qui-n-a-rien-de-low-cost.html

[SAU 12] SAUNIERE, J-C., LEROYER, S., «Innovation collaborative et propriété intellectuelle. Quelques bonnes pratiques », Etude INPI, 120p, 2012.

[SAV 16] SAVORnin, J-CH., Contract management : Outils et méthodes, Eds. EMS, Mai 2016.

[SER 14] SERVAJEAN-HILST, R., S'engager dans l'open innovation -Fondations, Technical Report, disponible à l'adresse https://www.researchgate.net/publication/269390473_S'engager_dans_l'Open_Innovation__Fondations_demarches_et_grandes_pratiques, Décembre 2014.

[TUR 01] TURNER, J.R., SIMISTER, S.J., «Project contract management and a theory of organization », International Journal of Project Management, Vol 19/8, 2001.

[VER 18] VERGES, E. (Dir.), Contrats sur la recherche et l'innovation, Dalloz, 908p., 2018.

[VON 86] VON HIPPEL, E., «Lead users : A source of novel product concepts », Management Science 32, $\mathrm{n}^{\circ}$, pp. 791-805, 1986. 